\title{
La centrale de pompage-turbinage de Guangzhou en République Populaire de Chine
}

\author{
par Jean-Jacques Smedts, Electricité de France, \\ Centre National d'Equipement Hydraulique (EDF-CNEH), \\ Chef de projet "Ingénierie »,
}

et Robert Faurel, Electricité de France,

Service de la Production Hydraulique (EDF-SPH),

Chef de projet "Formation-Exploitation ».

\section{La production d'électricité en Chine}

La Chine développe très rapidement ses moyens de production et de transport d'électricité : l'accroissement de la production est $+9 \%$ par an, et le parc de production a franchi en 1987 le cap des $100000 \mathrm{MW}$ installés pour atteindre $127000 \mathrm{MW}$ (dont près de $30 \%$ d'hydraulique) à fin 1989 (en France, $100300 \mathrm{MW}$ ) pour une production de $500 \mathrm{TWh} / \mathrm{an}$.

Cependant, le secteur de l'électricité présente des caractères particuliers, dans cet immense pays aux climats et reliefs très contrastés :

- Les distances sont trop grandes pour que l'ensemble du réseau soit interconnecté, et il existe 13 réseaux régionaux ou provinciaux. Les réseaux provinciaux concernent surtout des provinces éloignées géographiquement, à faible densité de population. Certains réseaux ont des échanges, mais d'importance limitée; certaines lignes d'échange sont en courant continu, qui permet le transit d'énergie mais pas l'assistance mutuelle au maintien de la tension et de la fréquence.

- Globalement, la production ne permet pas encore de satisfaire toute la demande. Les pointes potentielles de consommation sont donc supérieures aux pointes réelles.

- La production est assurée à $80 \%$ par le charbon et $20 \%$ par l'hydraulique. Le nucléaire est pour le moment négligeable. Les centrales à charbon ont des capacités de modulation de puissance limitées, et de plus leur rendement chute fortement lorsqu'elles fonctionnent à puissance réduite.

\section{The Guangzhou pumped storage power station,} in the People's Republic of China

The Guangzhou pumped storage power station, in South China, is with $1200 \mathrm{MW}$ in four reversible units the first large capacity pumped storage power station in China. It represents a technical innovation in this country, the hydro potential of which is enormous, and it is also an opportunity for a decisive progress in operation methods, the basis of which was up to now a very numerous staff. French engineering and industry are deeply involved in this project. Electricité de France-International, which exports consultancy and assistance services performed by all the Divisions of Electricité de France (EDF), provides technical assistance services to the chinese Owner (Guangzhou pumped Storage Power Station JVC) for design and construction, as well as for the training of operating staff and the operation management during the first two years of operation. A Consortium of french manufacturers, CEGELEC being the leader, supplies the whole electro-mechanical equipement of the project. EDF-International is also in charge of engineering and quality control services in the frame of this Consortium. 
Les réseaux ont donc besoin de moyens de production souples permettant de répondre rapidement aux variations de la consommation, prévues ou aléatoires.

L'hydraulique avec stockage est une réponse à ce besoin mais les ressources sont faibles dans les régions dont les consommations sont maximales, mise à part la région Centre traversée par le Yang-Tsé sur lequel a été construit le grand barrage de Gezhouba (2 $700 \mathrm{MW}$ ).

Cette situation a amené la Chine à s'intéresser au pompage-turbinage, qui, ne "consommant" pas d'eau, peut être réalisé en dehors de cours d'eau importants, et apporte au réseau à la fois :

- la souplesse d'adaptation aux variations de la consommation ;

- la rapidité d'intervention sur le réseau (de l'ordre de 3 min entre machines arrêtées et machines à pleine puissance);

- le secours en cas de défaillance passagère d'une centrale, ou d'une ligne qui en évacue la puissance ;

- une économie de combustible, les centrales à charbon pouvant être maintenues en permanence à leur rendement optimal puisque la modulation est faite par le pompageturbinage, et la réserve tournante en centrales thermiques (tournant à faible puissance) pouvant être diminuée: la rapidité d'intervention du pompage-turbinage permet de le considérer, même à l'arrêt, comme une réserve tournante.

Par contre, bien sûr, le rendement d'un cycle pompageturbinage est de l'ordre de $75 \%$ (il dépend des pertes de charge, donc des circuits hydrauliques d'amenée et de fuite). Il y a donc perte d'énergie, mais d'une part cette perte est au moins en partie compensée par des économies de combustible (charbon), d'autre part le bilan économique d'une centrale de pompage-turbinage de grande puissance, par rapport à d'autres moyens de production d'énergie de pointe (turbines à gaz par exemple), est souvent favorable, à condition qu'existent des sites bien adaptés par leur conditions naturelles (topographie, géologie) et par leur proximité du réseau de grand transport.

Ces conditions favorables existent dans beaucoup de régions de Chine, où des dénivellations de 300 à $600 \mathrm{~m}$, et même au-delà, peuvent être trouvées à des distances acceptables des grands réseaux : par exemple les sites de Ming Tombs près de Pékin, ou Tian Huang Ping près de Shanghai et Hangzhou, dans la province de Zhejiang. Sur ces deux sites, des travaux préparatoires ou des reconnaissances approfondies ont été faits. D'autres sites ont été reconnus, le total étant de l'ordre de $10000 \mathrm{MW}$.

Cependant, la Chine ne dispose jusqu'à maintenant que de groupes de pompage-turbinage de hauteur de chute et puissance modérées, installés sur des barrages-réservoirs en complément de groupes gravitaires classiques.

La centrale de Guangzhou (ou Cong Hua, nom d'une petite ville thermale proche du site), en construction depuis l'été 1988 à $100 \mathrm{~km}$ au Nord-est de la ville de Guangzhou (Canton) en Chine du Sud et dont le premier groupe doit être mis en service en novembre 1992, est la première réalisation de pompage-turbinage à grande échelle en Chine.

\section{Le réseau du Sud de la Chine}

La situation du réseau du Sud de la Chine, centré principalement sur les centres de consommation de la province de Guangdong au développement très rapide, est très favorable au pompage-turbinage, puisqu'outre des centrales au charbon, le réseau sera alimenté à partir de 1992-1993 par les $1800 \mathrm{MW}$ de la centrale nucléaire de Daya Bay, en construction avec une participation majoritaire de l'industrie et de l'ingénierie françaises (en particulier EDF, architecte industriel de cette réalisation). Le coût marginal de l'énergie nucléaire est très faible, et le pompage-turbinage permet de l'utiliser constamment en base, à la capacité maximale.

Une autre source d'alimentation à faible coût marginal est le transport d'énergie hydraulique depuis la province de Guangxi (projet en construction de Tian Shen Qiao). Les lignes sont très longues, et la gestion optimale est leur utilisation permanente à la capacité maximale. Cet apport est donc de l'énergie de base, non modulée.

Par ailleurs, les variations de la demande au cours de la journée sont assez fortes, le minimum étant voisin de la moitié du maximum.

\section{Le site de Guangzhou}

Le site retenu pour la construction de la centrale de pompage-turbinage a des caractéristiques naturelles favorables: possibilité de créer deux retenues distantes de moins de $4 \mathrm{~km}$ avec plus de $500 \mathrm{~m}$ de différence d'altitude (voir le profil en long, fig. I) - géologie favorable au creusement de grandes cavités souterraines (massif granitique, à bonne étanchéité) - apports naturels suffisants dans les retenues - proximité du nœud important du réseau $500 \mathrm{kV}$ constitué par la ville de Canton (Guangzhou).

\section{Les rôles de l'ingénierie et de l'industrie françaises}

Les études de faisabilité, faites par l'Institut de Projets Hydrauliques de la Province de Guangdong, ont été terminées en 1987. Dès ce stade, Electricité de FranceInternational est intervenue, en réalisant pendant l'été 1987 une " revue de projet " dont un certain nombre de conclusions ont été immédiatement retenues et incorporées au projet.

Après cette mise au point, un appel d'offres international a été lancé par la société créée pour être le Maître d'ouvrage (propriétaire et exploitant) de la centrale (la GPSJVC, Guangzhou Pumped Storage Power Station Joint Venture Corporation), pour la fourniture et la supervision de montage de l'ensemble des matériels électromécaniques. Une clause précisait que les groupements industriels consultés devaient s'assurer les services d'un ingénieur-conseil expérimenté en pompage-turbinage.

Compte tenu de l'importance de l'expérience française en pompage-turbinage (EDF a conçu et réalisé et exploite 
$4900 \mathrm{MW}$ de pompage-turbinage, ce qui représente $7 \%$ de la puissance mondiale dans ce domaine et place la France au $4^{\mathrm{c}}$ rang dans le monde), un groupement français constitué par CEGELEC (fourniture du contrôlecommande et d'équipements auxiliaires, pilotage du groupement) - Neyrpic (pompes-turbines et vannes) Alsthom-Jeumont (alternateurs-moteurs) - GECAlsthom (transformateurs et poste blindé à très haute tension) - SPIE-Batignolles (ponts roulants, auxiliaires électriques, câblages) a été consulté. Il a choisi EDF comme son ingénieur-conseil, pour les études d'ensemble, la définition détaillée des matériels, le contrôle de la qualité (fabrication et montage) et la coordination sur place des montages et essais. Son offre a abouti à un contrat signé en janvier 1989.

Parallèlement, la GPSJVC avait demandé à EDF une offre de prestations d'assistance technique complémentaires à celles prévues auprès des industriels : réalisation de certaines études - conseils à la réalisation, surtout en géologie, travaux souterrains, auscultation des ouvrages, liaisons entre génie civil et matériels, et planification du chantier - formation des exploitants - organisation de l'exploitation et encadrement des exploitants chinois pendant le démarrage et les deux premières années de fonctionnement. Il en est résulté un contrat, signé en janvier 1989, se prolongeant jusqu'en octobre 1994, soit un an après la mise en service industriel complète de la centrale. En particulier, le premier Directeur de la centrale sera un exploitant du Service de la Production Hydraulique (Groupe Régional Alpes) d'EDF, Les prestations d'ingénierie sont assurées par le Centre National d'Equipement Hydraulique d'EDF, installé depuis 1989 à SavoieTechnolac près de Chambéry.

La GPSJVC a confié à d'autres consultants certaines études particulières :

- Swedpower (Suède) : étude des phases de creusement et suivi géotechnique des grandes cavernes;

- Harza (USA) : calcul du répartiteur haute pression (voir ci-après).

\section{Le projet en cours de réalisation}

(voir figs. 1, 2, 3 et 4)

\section{Les retenues}

Les deux retenues, supérieure et inférieure, sont créées par des barrages de hauteur modérée:

- le barrage supérieur, $65 \mathrm{~m}$ de hauteur et $330 \mathrm{~m}$ de longueur en crête, est en enrochements compactés à masque amont en béton, mis en place par bandes de $12 \mathrm{~m}$ de largeur sans joints horizontaux ;

- le barrage inférieur, $42 \mathrm{~m}$ de hauteur et $150 \mathrm{~m}$ de longueur en crête, est en béton compacté au rouleau, avec un profil poids.

Les deux retenues ont la même capacité utile, 17 millions de $\mathrm{m}^{3}$, avec des marnages respectifs de $19,8 \mathrm{~m}$ et $12,4 \mathrm{~m}$, et des niveaux de retenue normale 816,8 et 287,4 . Les apports naturels sont nettement plus faibles pour la retenue supérieure : 6,6 millions de $\mathrm{m}^{3} / \mathrm{an}$, que pour la retenue inférieure : 17,2 millions de $\mathrm{m}^{3} / \mathrm{an}$.

\section{Le circuit hydraulique (voir fig. I)}

La conception du circuit hydraulique qui relie les retenues, entièrement en souterrain, est "un circuit unique pour quatre groupes ", ce qui diminue le coût des ouvrages et les pertes de charge, mais conduit à des galeries de grandes sections. C'est EDF, avec la participation de deux ingénieurs chinois, qui a étudié les comportements du circuit hydraulique et des groupes en transitoires normaux ou exceptionnels. Cette étude a en particulier permis le dimensionnement définitif des cheminées d'équilibre.

\section{A partir de la retenue supérieure on trouve:}

- Une prise d'eau pouvant entonner (ou rejeter, d'où un important convergent, ou divergent en pompage) un débit atteignant $280 \mathrm{~m}^{3} / \mathrm{s}$ à la chute minimale. Elle est équipée de panneaux de grilles puis se divise en deux pertuis équipés chacun d'une vanne-wagon à manœuvre lente (vannesbatardeaux).

- Une galerie d'amenée, diamètre $9 \mathrm{~m}$, longueur $860 \mathrm{~m}$, pente $5,7 \%$.

- A son extrémité, une cheminée d'équilibre à fût de $14 \mathrm{~m}$ de diamètre, reliée à la galerie par un puits de $8,5 \mathrm{~m}$ de diamètre et un diaphragme de $5,6 \mathrm{~m}$, et comportant un bassin d'expansion supérieur de $25 \mathrm{~m}$ de diamètre.

- Deux puits inclinés successifs, diamètre $8,5 \mathrm{~m}$, longueurs $400 \mathrm{~m}$ et $300 \mathrm{~m}$, pente $119 \%$, reliés par un court tronçon horizontal. Ce tronçon, réalisé depuis une fenêtre d'accès, permet d'éviter la grande longueur d'un puits unique, et de diminuer la durée des travaux par une réalisation en parallèle des deux puits. Il faut remarquer que le creusement est fait à l'explosif, avec un avant-puits de $2 \mathrm{~m} \times 2 \mathrm{~m}$ servant ensuite à l'évacuation des déblais de l'élargissement au diamètre définitif, fait de haut en bas; cette technique impose une forte pente (au moins $80 \%$ ); les réalisations françaises les plus récentes ont au contraire été creusées au tunnelier, avec des pentes plus faibles $(56 \%$ à Grand Maison, $43 \%$ à Super Bissorte), sans rupture de pente, ce qui permet un circuit plus court et moins de coudes, de réalisation toujours coûteuse. A Guangzhou les puits sont revêtus en béton armé, sans blindage. Le contact entre le revêtement et le rocher est injecté, et les injections sont prolongées dans la couronne de rocher décomprimé par le creusement pour lui redonner sa cohésion.

- Une galerie haute pression horizontale de $230 \mathrm{~m}$, dont le diamètre se réduit de $8,5 \mathrm{~m}$ à $8 \mathrm{~m}$, et qui se termine par un répartiteur alimentant les quatre rameaux (diamètre 3,5 m) des groupes. La conception du revêtement est identique à celle des puits inclinés, mais la pression d'injection est portée à la pression intérieure de service de la galerie, pour assurer une précontrainte dans les parties où le gradient hydraulique en cas de fissures débouchant sur une surface libre serait trop élevé.

- Les rameaux haute pression des groupes, diamètre $3,5 \mathrm{~m}$, longueur $150 \mathrm{~m}$, blindés en acier à haute limite 


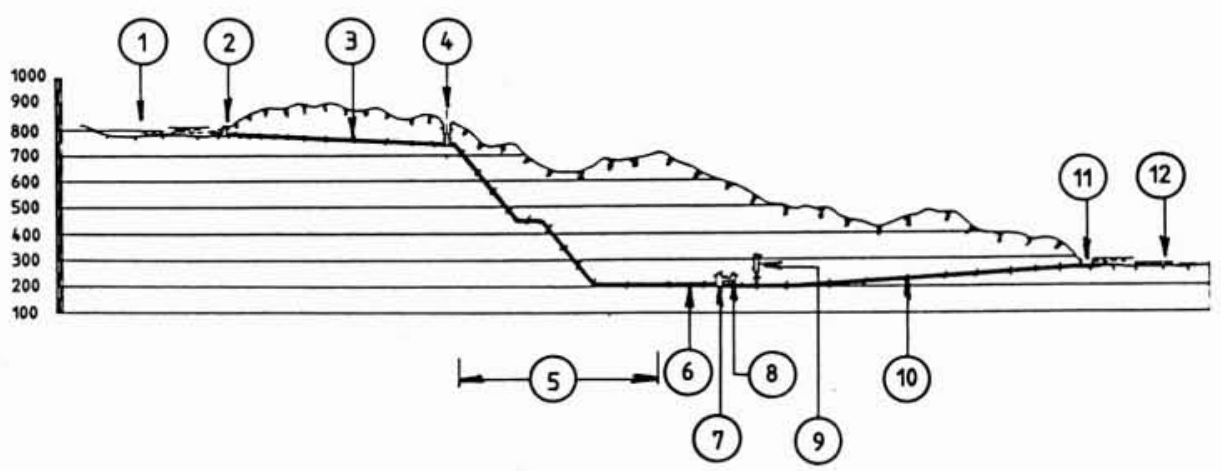

1. Profil en long général des ouvrages.

Longitudinal section of the scheme.

1. Retenue supérieure

2. Prise d'eau supérieure

Upper reservoir

3. Galerie d'amenée

4. Cheminée d'équilibre supérieure

5. Puits inclinés et galerie haute pression ... Upper intake

6. Répartiteur et rameaux blindés haute pression

Ueadrace (pressure) tunnel

7. Salle des machines

8. Salle des transformateurs et du poste blindé

Upper surge shaft

9. Cheminées d'équilibre inférieures

10. Galerie de fuite (basse pression)

11. Prise d'eau inférieure

High pressure inclined shafts and tunnel

High pressure manifold and steel lined branches

Powerhouse

Transformers and G.I.S. hall

Lower surge shafts

Tailrace (low pressure) tunnel

12. Retenue inférieure

Lower intake

Lower reservoir

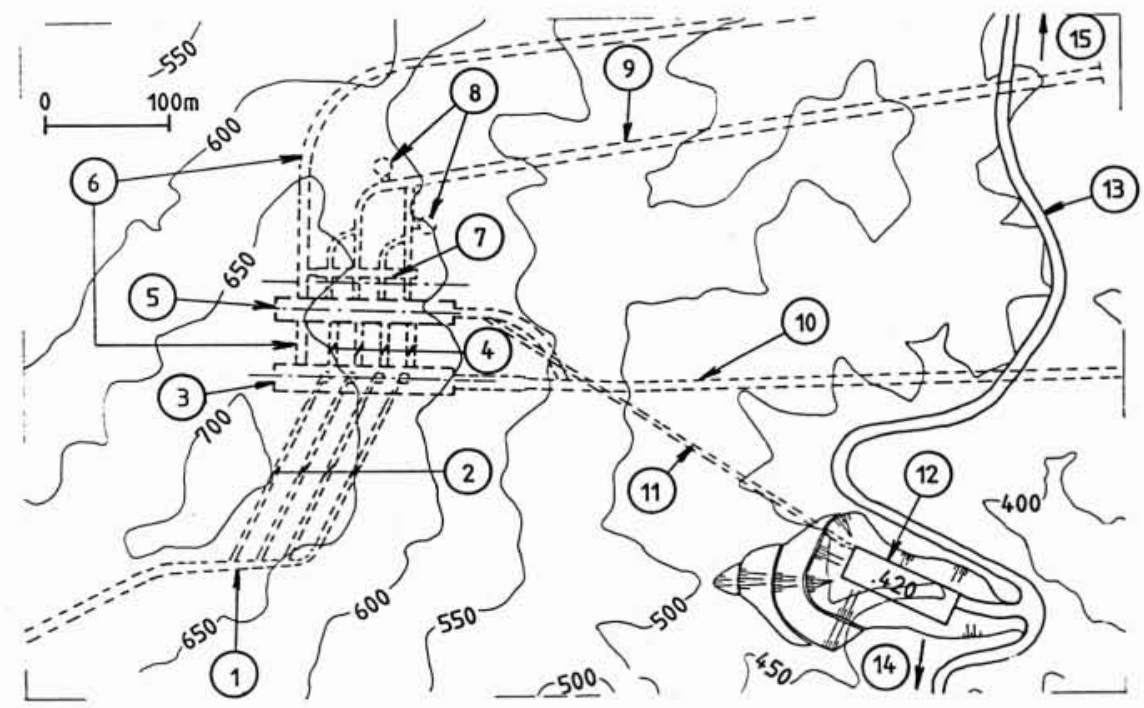

2. Vue en plan des ouvrages au voisinage de la centrale.

Plan view of the works near the powerhouse.

1. Répartiteur haute pression

2. Rameaux blindés haute pression

3. Salle des machines

4. Galeries des barres MT

5. Salle des transformateurs et du poste blindé

6. Galerie d'accès

7. Galerie des vannes basse pression

8. Cheminées d'équilibre inférieures

9. Galerie de fuite (basse pression)

10. Galerie de ventilation

11. Galerie des câbles $500 \mathrm{kV}$

12. Poste aéro-souterrain $500 \mathrm{kV}$

13. Route d'accès aux ouvrages supérieurs

14. Vers la retenue inférieure

15. Vers la retenue supérieure

Note: Seuls les ouvrages principaux sont représentés

Only the main parts are drawn.
High pressure manifold

High pressure steel-lined branches

Powerhouse

MV busbars tunnels

Transformers and G.I.S. hall

Access tunnel

Tailrace gates cavern

Lower surge shafts

Tailrace (low pressure) tunnel

Ventilation tunnel

$500 \mathrm{kV}$ cables tunnel

Outdoor $500 \mathrm{kV}$ switchyard

Access road to the upper parts of the scheme

to lower reservoir

to upper reservoir 


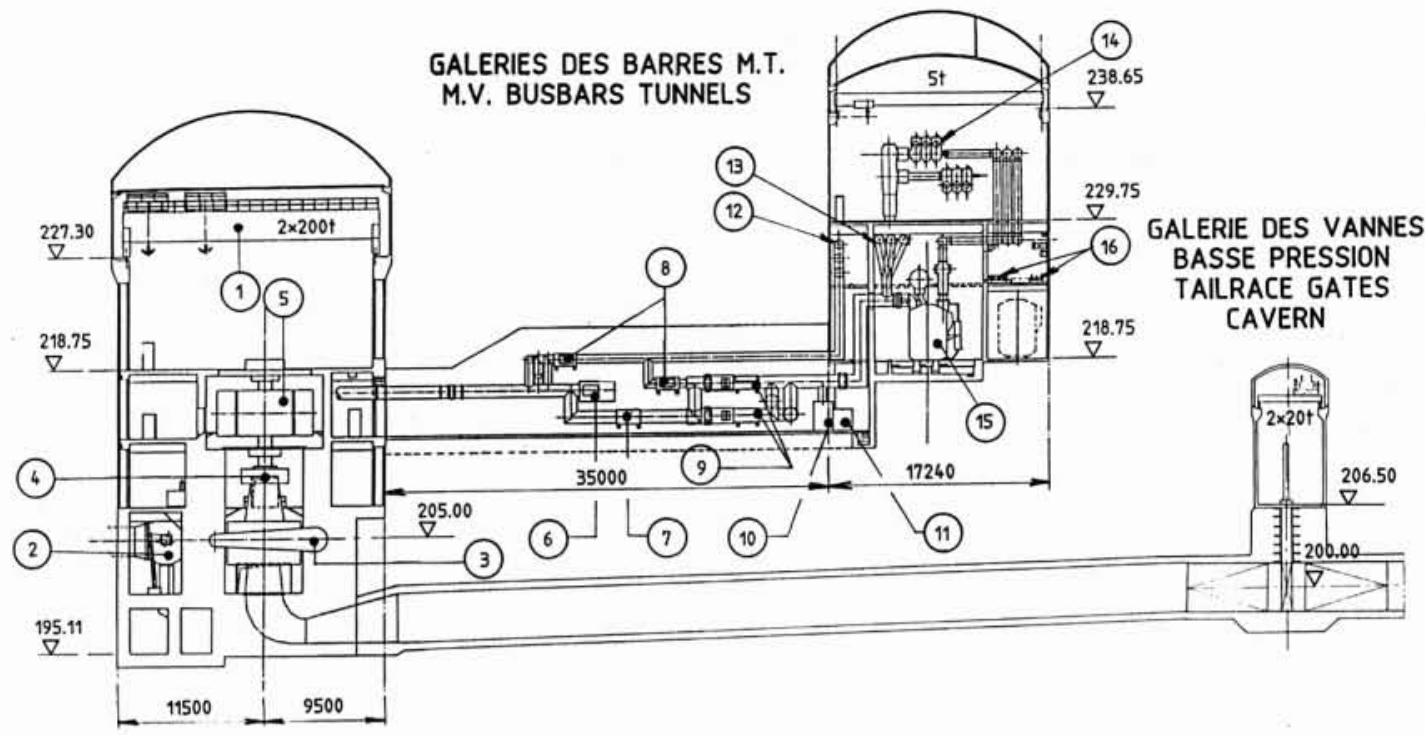

3. Coupe transversale de la centrale.

Cross section of the powerhouse.

1. Pont roulant principal

Main overhead crane

2. Robinet sphérique $(\varnothing 2,21 \mathrm{~m})$

Spherical valve $(\varnothing 2.21 \mathrm{~m})$

3. Bâche spirale

Spiral case

4. Pivot

Thrust-bearing

5. Moteur-alternateur

Motor-generator

6. Sectionneur de freinage électrique

Electric braking switch

7. Disjoncteur de groupe

MV circuit breaker

8. Sectionneurs de démarrage

Starting switches

9. Sectionneur inverseur de phases

Phases reversing switch

10. Transformateur d'excitation

Excitation transformer

11. Transformateur de tension (protections)

Potential transformer (protections)

12. Jeu de barres de démarrage

Starting busbars

13. Jeu de barres d'auxiliaires

Station service busbars

14. Poste blindé SF6 $500 \mathrm{kV}$

$500 \mathrm{kV}$ Gas Insulated Switchyard (GIS

15. Transformateur principal

Main transformer

16. Câbles $500 \mathrm{kV}$

$500 \mathrm{kV}$ cables

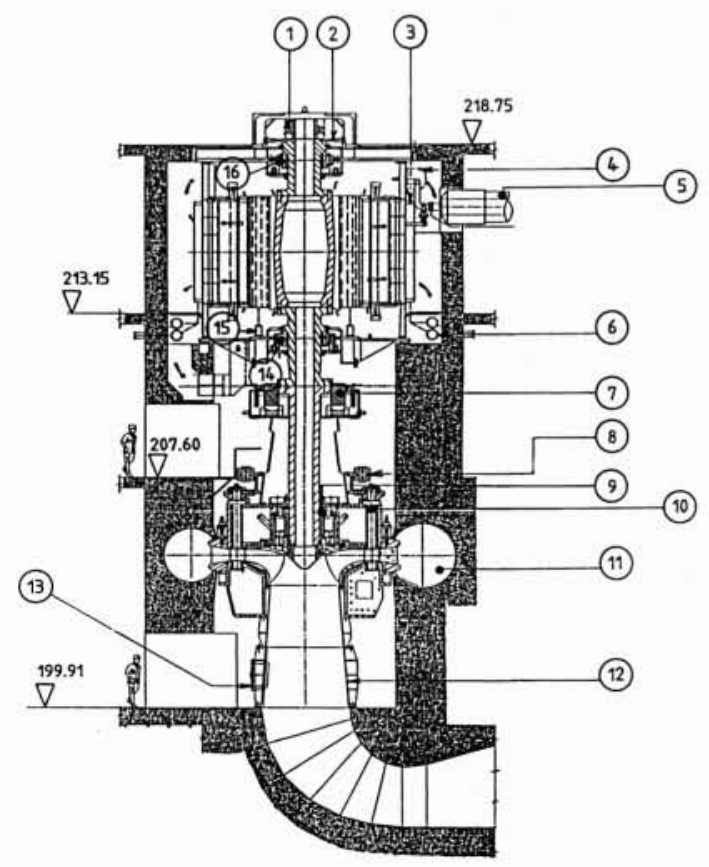

4. Coupe transversale sur un groupe Cross section of a unit.

1. Excitation

Excitation

2. Frein à disque

Disc brake

3. Réfrigérant eau/air

Water/air cooler

4. Ventilateur

Ventilator

5. Jeu de barres MT (18 kV)

MV (18 kV) busbars

6. Conduites d'eau de réfrigération

Cooling-water main pipes

7. Pivot

Thrust-bearing

8. Cercle de vannage

Wicket-gates operating ring

9. Palier-guide de la pompe-turbine

Pump-turbine guide-bearing

10. Directrices

11. Bâche spirale

Wicket-gate.

12. Cône d'aspirateur

Spiral case

13. Porte de visite de la roue ............ Access door for runner inspection

14. Palier inférieur du moteur-alternateur ......... Motor-generator lower guide-bearing

15. Vérins de levage du rotor ......................... Rotor lifting jacks

16. Palier supérieur du moteur-alternateur ....... Motor-generator upper guide-bearing 
d'élasticité. Le blindage est bloqué au rocher par bétonnage et injections.

- La centrale souterraine abritant les groupes, y compris leurs robinets sphériques de protection côté haute pression (diamètre 2,21 m).

- Les rameaux de fuite basse pression des groupes, diamètre $4 \mathrm{~m}$, qui se rassemblent d'abord deux par deux puis en une galerie de fuite unique, diamètre $8 \mathrm{~m}$. Chaque rameau est équipé d'une vanne plate sous carter, protégeant le groupe côté basse pression.

- Les deux cheminées d'équilibre inférieures, puits «borgnes " de $55 \mathrm{~m}$ de hauteur et $14 \mathrm{~m}$ de diamètre, reliées chacune à un rameau de fuite regroupant ceux de deux groupes par un puits de diamètre $5,6 \mathrm{~m}$ et un diaphragme de $4 \mathrm{~m}$. Les sommets des puits sont reliés par une galerie horizontale fonctionnant en chambre d'expansion supérieure, alimentée en air par une galerie inclinée débouchant à l'air libre.

- La galerie de fuite, dont le diamètre passe rapidement à $9 \mathrm{~m}$, longueur $1320 \mathrm{~m}$, pente $5,3 \%$ (montante de la centrale vers la retenue inférieure).

- La prise d'eau inférieure, identique à la prise d'eau supérieure.

\section{Les équipements électro-mécaniques}

Les principales caractéristiques des quatre groupes sont (voir fig. 4) :

- Pompe-turbine mono-étage de $306 \mathrm{MW}$ à 500 tours par minute sous $511 \mathrm{~m}$ de chute nette en turbine, débit nominal $66,4 \mathrm{~m}^{3} / \mathrm{s}$, et $317 \mathrm{MW}$ pour refouler en pompe $56,3 \mathrm{~m}^{3} / \mathrm{s}$ sous $533 \mathrm{~m}$ de hauteur nette. L'enfoncement des roues est de $70 \mathrm{~m}$ (axe de la bâche spirale à l'altitude 205). Les rendements, garantis et vérifiés sur modèle, sont élevés : supérieurs à $92 \%$ aussi bien pompe qu'en turbine.

- Alternateur-moteur à pôles saillants, 333 MVA (363 MVA en surcharge continue), sous cos phi 0,9 en alternateur et 0,95 à 1 en moteur, tension $18 \mathrm{kV}$. La réfrigération est à air, par ventilateurs motorisés indépendants de la rotation du groupe. Le freinage est assuré d'abord (entre $50 \%$ et $5 \%$ de la vitesse nominale) électriquement par mise en court-circuit du stator, puis terminé par un frein à disque monté sur l'arbre au-dessus du rotor. Il n'y a donc pas de piste de freinage sous la jante du rotor, et les vérins ne sont utilisés que pour le soulèvement du rotor à l'arrêt, pour permettre des inverventions sur le pivot.

- Le pivot, calculé pour une poussée maximale transitoire de 980 tonnes, est installé au-dessus du flasque supérieur de la pompe-turbine. Ses 10 patins sont montés sur soufflets hydrauliques reliés entre eux pour équilibrer les charges sur chaque patin. Une pompe injecte de l'huile entre glace et patins pendant les phases de démarrage et d'arrêt.

Le démontage de la roue de pompe-turbine se fait par en dessous, sans démontage de l'alternateur-moteur, donc sans nécessité de régler la ligne d'arbre à chaque remontage. Pour la sortie de la roue, le flasque inférieur de la pompe-turbine et la partie supérieure de l'aspirateur, conique, se démontent. Cette disposition, adoptée presque systématiquement par EDF, permet un gain de temps considérable sur démontage et remontage de roue.

L'énergie est évacuée de l'alternateur par des gaines coaxiales en $18 \mathrm{kV}$, qui empruntent des galeries (une par groupe) perpendiculaires à la centrale. Ces galeries abritent les disjoncteurs de groupes et les sectionneurs à 5 pôles (inverseurs de phases) pour le changement de sens de rotation (de pompe en turbine et vice versa), ainsi que les sectionneurs de freinage électrique et de démarrage, et les transformateurs d'excitation.

Les gaines rejoignent ensuite les transformateurs, triphasés $18 / 525 \mathrm{kV}$ (un par groupe), réfrigérés par des hydroréfrigérants. Les transformateurs sont placés dans une galerie parallèle à la centrale, et abritant également le poste $525 \mathrm{kV}$, au-dessus des transformateurs.

Le poste, blindé sous hexafluorure de soufre (SF6) pour réduire les dimensions, est constitué d'un jeu de barres " circulaire" ou "à quatre sommets" (" ringbus") avec deux départs d'évacuation d'énergie. Le jeu de barres est coupé par quatre disjoncteurs. Au prix d'une diminution de la souplesse d'exploitation, acceptée par le Maître d'ouvrage, cette disposition est la plus économique. EDF, pour ses propres centrales, utilise la disposition à double jeu de barres, qui offre une souplesse d'exploitation très supérieure. L'énergie est évacuée par câbles à huile $525 \mathrm{kV}$ (section de cuivre $1600 \mathrm{~mm}^{2}$ ) vers un poste de départ aéro-souterrain (deux départs de lignes aériennes $525 \mathrm{kV}$ vers des postes proches de la ville de Canton). Les six câbles monophasés sont posés dans une galerie de $400 \mathrm{~m}$ de longueur à $55 \%$ de pente, ventilée et équipée d'une protection contre l'incendie. La dénivellation entre têtes de câbles hautes et basses a été limitée à moins de $200 \mathrm{~m}$ pour éviter d'équiper les câbles de boîtes de jonction limitant la pression d'huile.

Le démarrage des groupes en pompe est assuré par alimentation à fréquence variable fournie par un convertisseur statique de fréquence de 27 MVA, commun aux quatre groupes, fonctionnant à $4,2 \mathrm{kV}$ pour diminuer le coût et l'encombrement des blocs de thyristors. Avant le démarrage, la roue de pompe-turbine est dénoyée par injection d'air comprimé dans la bâche spirale. Cela évite d'avoir à fournir la puissance de barbotage de la roue dans l'eau, qui est très élevée. Un jeu de barres de démarrage relie les sorties $18 \mathrm{kV}$ des quatre groupes. Un démarrage en dos à dos, par couplage électrique entre un groupe "lanceur " démarrant en turbine et un groupe "lancé " démarrant en pompe, est prévu en secours en cas d'incident sur le convertisseur de fréquence.

Les groupes peuvent également fonctionner en compensateur synchrone pour le réglage de tension du réseau. La roue de pompe-turbine est alors maintenue dans l'air.

Le fonctionnement nécessite évidemment un grand nombre d'équipements auxiliaires, notamment :

- Les ponts roulants, parmi lesquels ceux de la salle des machines : pont principal à deux chariots de $200 \mathrm{t}$ chacun, portée $19,5 \mathrm{~m}$, et pont auxiliaire (manutentions courantes) de $30 \mathrm{t}$.

- Les pompes de réfrigération et les pompes d'exhaure. Les eaux d'exhaure sont rejetées dans la retenue inférieure 
par deux conduites de $600 \mathrm{~mm}$ noyées dans le béton de revêtement de la galerie de fuite.

- Les équipements d'alimentation électrique des auxiliaires (alternatif et continu), de contrôle-commande (entièrement numérique), les compresseurs, la climatisation et ventilation, les télécommunications, regroupés dans un bâtiment des auxiliaires à une extrémité de la centrale.

La centrale est reliée à l'air libre par deux galeries :

- La galerie d'accès, longue de $1550 \mathrm{~m}$, pente $5,4 \%$, qui dessert la plage de manutention et montage des matériels, au niveau supérieur des groupes. La plage de montage est à l'extrémité de la salle des machines opposée au bâtiment des auxiliaires.

- La galerie de ventilation, plus courte : $1090 \mathrm{~m}$, pente $8,2 \%$, qui part du niveau supérieur du bâtiment des auxiliaires.

\section{Le déroulement des travaux}

Les travaux ont commencé en juillet 1988, dans des conditions difficiles dues au mauvais état de la route de $60 \mathrm{~km}$ reliant Conghua (à $70 \mathrm{~km}$ de Canton) au site.

Les premiers travaux entrepris ont été à la fois le creusement de la galerie de ventilation, qui était le plus court chemin pour atteindre l'emplacement de la centrale souterraine, et la construction de la route de $13 \mathrm{~km}$ menant à la retenue supérieure, dans un terrain au relief extrêmement accidenté. Cette route était indispensable pour un démarrage rapide des travaux du barrage supérieur: compte tenu des deux saisons des pluies nécessaires pour remplir la retenue jusqu'au niveau minimal d'exploitation, la construction du barrage supérieur devait permettre le début du remplissage avant l'été 1991.

La majeure partie des travaux, et en particulier tous les travaux souterrains, sont exécutés par l'entreprise chinoise " $14^{\mathrm{e}}$ Bureau de Construction des Ministères de l'Energie et des Ressources Hydrauliques ".

En mars 1991, la situation est la suivante sur les principaux ouvrages :

- Le masque amont du barrage supérieur, construit jusqu'à une cote intermédiaire, est en cours de bétonnage en vue d'un début de remplissage de la retenue en avril. La construction du barrage se poursuivra pendant le remplissage.

- La prise d'eau supérieure se termine.

- La galerie d'amenée et les puits inclinés sont en cours de creusement.

- Le creusement de la centrale souterraine est terminé (dimensions maximales de l'excavation: longueur $146 \mathrm{~m}$, largeur $22 \mathrm{~m}$, hauteur $45 \mathrm{~m}$ ), les ponts roulants sont montés et le coude d'aspirateur du premier groupe est en place et en cours de soudage.

- La galerie de fuite est en cours de bétonnage.

- Les fouilles de la prise d'eau inférieure sont faites.

- Le décapage de la fondation du barrage inférieur se termine.

- Autour de la retenue inférieure, de nouveaux groupes d'habitations ont été construits pour reloger les 1200 personnes dont les villages seront submergés.

- Le bâtiment de logements et bureaux d'exploitation est terminé et utilisé pour les besoins de la direction des travaux.

Parallèlement, la route depuis Conghua a été considérablement améliorée, avec un tracé nouveau, beaucoup plus court, sur une grande partie du parcours. Il suffit maintenant de deux heures de voitures pour venir de Canton. Les ponts anciens sont en cours de renforcement pour le passage des matériels. Les masses indivisibles les plus lourdes (les robinets sphériques, $120 \mathrm{t}$, et les transformateurs, $200 \mathrm{t}$ ) nécessiteront des itinéraires spéciaux évitant certains points du parcours.

Les montages des matériels sont faits par la branche spécialisée de l'entreprise chinoise " $14^{\mathrm{e}}$ Bureau » chargée du génie civil, sous la supervision d'ingénieurs et techniciens du consortium industriel français.

Les superviseurs, parmi lesquels un ingénieur d'EDF$\mathrm{CNEH}$ assure des contrôles de qualité et la coordination des montages et essais, habitent le «village des expatriés » à Conghua, construit par GPSJVC. Les premiers s'y sont installés en janvier 1991.

La fabrication de certaines parties de matériels hydrauliques a été sous-traitée par NEYRPIC à des sociétés chinoises spécialisées : blindages, coudes d'aspirateurs (les deux premiers ont toutefois été fabriqués en France pour assurer le respect du programme des travaux), tabliers et carters des vannes basse pression, cercles de vannage, flasques des pompes-turbines.

Les ponts roulants circulent sur des poutres en béton armé ancrées dans les parois de la salle des machines, sans poteaux supports. Cela a permis le bétonnage des poutres, la pose des voies de roulement et même le montage des ponts avant la fin du creusement des parties basses de la centrale, gagnant ainsi plusieurs semaines sur le délai de début de montage.

La mise en service du premier groupe est prévue en novembre 1992. Les trois autres groupes suivront avec un pas de trois mois. Le circuit hydraulique commun aux quatre groupes nécessite que toutes les vannes basse pression et robinets sphériques soient en place dès la mise en eau des parties basse pression et haute pression du circuit. Le programme de fabrication tient évidemment compte de cet impératif.

\section{L'exploitation de la centrale}

La centrale est un élément primordial dans l'exploitation du réseau $500 \mathrm{kV}$ du Sud de la Chine, auquel est relié le réseau $400 \mathrm{kV}$ de Hong-Kong.

Elle est prévue pour être télécommandée depuis le dispatching de réseau situé à Canton. Les liaisons de télécommunications pour cette télécommande sont assurées par deux voies: courants porteurs en ligne, et liaison hertzienne par ondes courtes.

Cependant, pendant les premières années de fonctionnement, la centrale sera exploitée avec un service de quart sur place : depuis la salle de commande, l'opérateur dispo- 
sera de l'ensemble des informations et des commandes pour adapter le fonctionnement (nombre de groupes en service, puissance de chaque groupe en turbine) à la puissance demandée par le réseau; le calculateur de contrôlecommande lui donnera les indications nécessaires pour assurer en permanence la configuration optimale.

L'organisation de l'exploitation de la centrale, étudiée conjointement par GPSJVC et EDF, est proche de celle qui est pratiquée par EDF pour ses propres centrales. En dehors du technicien assurant la présence permanente dans la salle de commande, le personnel travaillera uniquement la journée, pour les contrôles et la maintenance des installations.

Un système d'astreinte, hiérarchisée suivant la nature des alarmes, est mis en place : en cas d'anomalie sur un matériel en dehors des heures de travail, un Diffuseur d'Alarme Parlée envoie automatiquement un message préenregistré (avec la nature résumée de l'anomalie) au poste téléphonique où on peut atteindre le technicien qui est en « astreinte » (veille technique). Si l'anomalie le nécessite, le technicien appelle un ingénieur qui est lui aussi en astreinte, pas l'intermédiaire du Diffuseur d'Alarme Parlée qui fait automatiquement la recherche.

Pour assurer la continuité de fonctionnement du dispositif, un réseau radio couvrira toute l'étendue du site des ouvrages et la route entre le site et les logements de Conghua ; les véhicules peuvent recevoir les appels. En outre un système de recherche de personnes sur le site signalera les appels, y compris dans les ouvrages souterrains où une présence de personnel est habituelle.

Il est prévu de pouvoir, depuis la centrale, faire les mesures d'auscultation les plus importantes pour la vérification de la sécurité des barrages et de la partie inférieure des ouvrages haute pression, et d'analyser immédiatement ces mesures pour en vérifier le caractère normal, par un logiciel incorporé au système corrigeant les mesures de l'influence des principaux paramètres de variation : cotes des plans d'eau et température.

Pour assurer la pleine sécurité de l'exploitation, les cotes des plans d'eau supérieur et inférieur et la position des vannes des prises d'eau sont transmises au calculateur de contrôle-commande par deux voies séparées, l'une assurant le secours de l'autre : il est en effet impératif d'éviter aux barrages tout déversement aux barrages par pompage ou turbinage au-delà du niveau de retenue normale (les débits ainsi déversés pourraient être supérieurs aux crues naturelles des cours d'eau barrés et être ainsi dangereux pour la population des vallées), et d'éviter également, pour la sécurité des installations, tout fonctionnement si les vannes de prise d'eau ne sont pas totalement ouvertes.

L'ensemble de ces dispositions, mises au point et éprouvées dans les propres installations d'EDF, permettra d'assurer l'exploitation de la centrale avec moins de 70 personnes, alors que la reconduction des méthodes employées jusqu'ici en Chine en aurait nécessité environ dix fois plus. Parmi les premiers cadres d'exploitation pleinement intégrés au personnel, sept ingénieurs et techniciens spécialisés du Service de la Production Hydraulique d'EDF, dont le premier Directeur de la centrale. Ils arriveront à partir de l'été 1991, période des premiers montages de parties mécaniques. La période des montages et des essais sera mise à profit pour familiariser le personnel chinois avec les équipements : utilisation, fonctionnement, entretien, méthodes de montage et démontage.

L'intervention des exploitants d'EDF, en nombre réduit progressivement à deux, s'étendra jusqu'en octobre 1994.

Les méthodes d'entretien préventif mises au point par EDF (MECEP: Méthode de Contrôles et d'Entretiens Préparés), basées sur la mesure de l'évolution de certains paramètres clés des matériels et la réparation avant que cette évolution n'accroisse trop la probabilité de défaillance, seront appliquées à la centrale de Guangzhou.

\section{La formation des exploitants}

L'acquisition de ces méthodes par les futurs exploitants chinois, précédée de celle des connaissances particulières à l'exploitation des centrales de pompage-turbinage par rapport à celle des centrales hydroélectriques classiques, imposait une formation approfondie.

Cette formation, dont le programme a été mis au point par EDF pour les différentes spécialités techniques (conduite en temps réel, entretien mécanique, électricité, électronique, informatique...) à partir de ses propres formations, a été assurée, entièrement en langue anglaise, par des formateurs du Centre de La Pérollière près de Lyon associés aux futurs exploitants français de la centrale : ces derniers ont ainsi travaillé très tôt côte à côte avec leurs futurs collègues chinois, ce qui est évidemment très favorable à la compréhension mutuelle des méthodes et à la cohésion des équipes. 40 ingénieurs et techniciens chinois d'âges et d'expériences très divers, certains à peine sortis de l'université, ont suivi en France une formation de plusieurs mois, à La Pérollière et dans des centrales de pompage-turbinage. Ils avaient auparavant été sélectionnés en Chine par des tests de connaissances techniques mis au point par EDF.

Cette formation a été ou sera pour la plupart des stagiaires complétée par un séjour de familiarisation avec les matériels, chez les constructeurs français.

\section{Vers le doublement et un record mondial}

La disposition des ouvrages permet, à partir des mêmes retenues supérieure et inférieure, de réaliser un deuxième équipement de pompage-turbinage identique au premier.

Le circuit hydraulique serait parallèle au premier, et de même conception. La salle des machines serait dans le prolongement de la première à une distance suffisante pour que les ébranlements dus au creusement ne gênent pas le fonctionnement des quatre premiers groupes. La galerie d'accès, commune aux deux salles des machines, permettrait un démarrage très rapide du creusement.

A l'origine il était prévu de surélever les barrages supérieur et inférieur (surélévations respectives : $7 \mathrm{~m}$ et $4,4 \mathrm{~m}$ ) au moment de la réalisation de cette deuxième étape, pour 
créer les volumes d'eau supplémentaires correspondants. Il est apparu plus économique de construire les barrages à la cote finale dès la première étape.

En effet GPSJVC envisage maintenant de réaliser la deuxième étape à court terme, pour une mise en service avant l'an 2000. Dans ce but, les deux prises d'eau de la deuxième étape sont construites dès l'étape actuelle.

Lorsque les deux centrales seront en service, Guangzhou détiendra avec $2400 \mathrm{MW}$ le record mondial de puissance installée en pompage-turbinage. Et la République Populaire de Chine ne compte pas en rester là : les projets sont nombreux, les sites sont de qualité et le Comité Chinois de
l'Electricité associé à la Société Chinoise de l'Ingénierie Hydroélectrique ont organisé à Pékin en octobre 1990 un «Symposium international sur le développement du pompage-turbinage » qui a montré l'intérêt porté à cette technique par le Ministère Chinois de l'Energie.

Electricité de France-International est prête à poursuivre sur d'autres projets de pompage-turbinage la collaboration avec le Ministère Chinois de l'Energie, commencée dès 1985 par des échanges techniques et concrétisée par le bon déroulement du projet de Guangzhou.

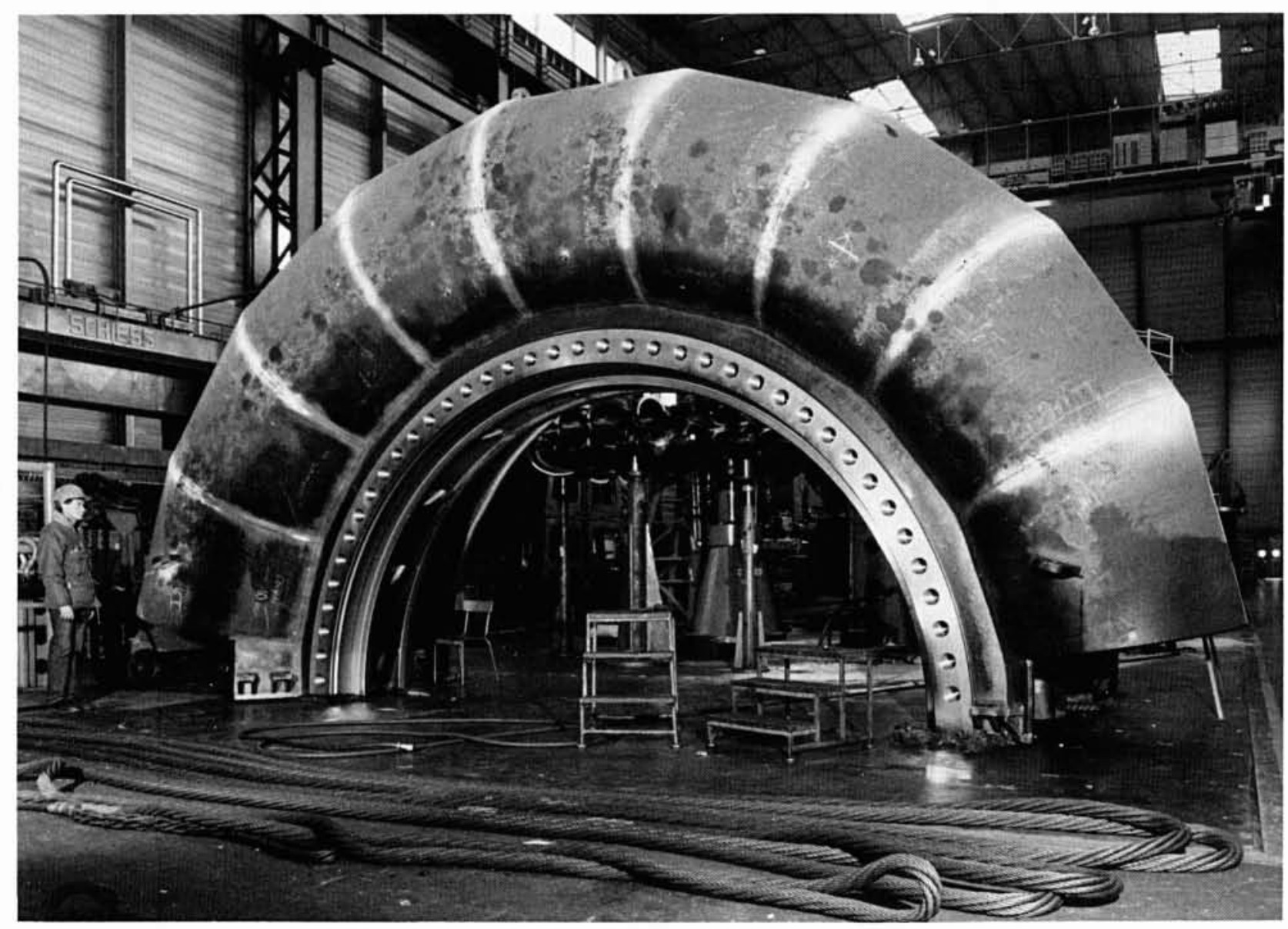

Demi-bâche spirale, avec les avant-directrices, en fin de fabrication chez NEYRPIC (Grenoble).

Half of the spiral case, with guide-vanes, at the end of manufacture in NEYRPIC workshop (Grenoble). 